\title{
A Normal Form Object-Oriented Entity Relationship Diagram
}

\author{
Tok Wang LING and Pit Koon TEO \\ Department of Information Systems and Computer Science \\ National University of Singapore
}

\begin{abstract}
A normal form object-oriented entity relationship (OOER) diagram is presented to address a set of $O O$ data modelling issues, viz. the inability to judge the quality of an $O O$ schema, the presence of inheritance conflicts in ISA hierarchies, the lack of explicit support for difierent relationship types (m-n, $n$-ary, recursive etc.) in some $O O$ data models and the lack of general and flexible support for views. Three approaches are described to translate good quality $O O$ schemas from a normal form OOER diagram. The first approach translates $0 O$ schemas whose underlying 00 data models support the notion of relationship. There are no undesirable redundancies in the translated schemas. The second approach provides for $\mathrm{OO}$ data models that do not support the notion of relationship. Some redundancies may arise because of $\mathbf{m}-\mathbf{n}$, $\mathbf{n}$-ary relationships, but these can be controlled. The third approach treats each OO schema as an external schema of a conceptual schema represented by a normal form OOER diagram. Redundancies may exist at the external schema level, but no redundancies exist at the conceptual schema level.
\end{abstract}

\section{Introduction}

The tremendous interest in the object-oriented $(00)$ approach has exposed a number of inadequacies [20] which are now widely recognised, e.g. lack of a formal foundation, a navigational, as opposed to a declarative interface, lack of a standard query language etc. Some of these issues have been addressed, but many remain unresolved. In this paper, we focus on a set of $O O$ data modelling issues which can be resolved by leveraging techniques $[14,15,16]$ in entity relationship (ER) data modelling[6]. These issues include (1) the inability to judge the quality of an 00 schema design, (2) the presence of inheritance conflicts in class hierarchies, (3) the lack of explicit support for different relationship types ( $m-n, n-a r y$, recursive etc.) in some $O O$ data models and (4) the lack of general and flexible support for views.

To address these issues, we introduce the notion of a normal form OOER diagram. A normal form OOER diagram extends the classical ER diagram[6] in three ways. First, it incorporates the behavioural notions of methods and derived attributes. The additions of these active components and associated semantics for inheritance and complex objects to an ER diagram produce an OOER diagram. Second, the structural properties of the OOER diagram, which are in fact those of an ER diagram, are in normal form. This guarantees that 
relations generated from a normal form OOER diagram are either in 3NF or 5NF. A normal form OOER diagram does not have undesirable redundancies. It allows the quality of a schema based on it to be judged. Third, it does not have any inheritance conflicts in its ISA hierarchies. In [19], we have proposed a complete algorithm which resolves inheritance conflicts in ISA hierarchies.

In this paper, we describe the translation of $O O$ schemas from a normal form OOER diagram. Each entity type can be translated directly into a class, but, generally, three approaches can be adopted to handle the translations for relationship sets. The first two approaches depend on whether the underlying $O O$ data model for a translated $O O$ schema provides explicit support for the notion of relationship. If the notion of relationship is supported, then each $\mathbf{m}-\mathbf{n}, \mathbf{n}$-ary or recursive relationship set can be translated into a class in the $O O$ schema. There are no undesirable redundancies in the translated schemas. Otherwise, an embedded, inter-class reference is used to link classes implicitly. In this case, some redundancies may arise because of $\mathrm{m}$-n and n-ary relationships, but these can be controlled. The third approach is a flexible view mechanism that treats each $O O$ schema as a view of a normal form OOER diagram. We propose a 3level ER-based schema architecture with the conceptual schema represented by a normal form OOER diagram. Each $0 O$ schema is treated as an external schema that is generated from the conceptual schema by using a set of mapping rules. In [18], mapping rules have been defined to generate 02 external schemas from an OOER diagram. The same rules can be used to generate $\mathrm{O} 2$ external schemas from a normal form OOER diagram. Distinct sets of mapping rules[15, 16] can be used to generate hierarchical, network, ER diagrams, and nested relations external schemas from a normal form OOER diagram. This approach is flexible in the sense that users can choose to view the conceptual schema in ways that they wish to and are comfortable with. The advantages of these data models (OO, hierarchical, network, etc.) are preserved at the external schema level.

Section 2 discusses the motivation behind this paper. Section 3 introduces the concept of an OOER diagram. In Section 4, the normal form OOER diagram is defined. Section 5 discusses the steps to transform an OOER diagram into a normal form OOER diagram. Section 6 discusses the generation of $O O$ schemas from a normal form OOER diagram. Section 7 concludes the paper.

\section{Motivation}

This paper is motivated by the following observations. First, it is difficult to judge the quality of an $O O$ database schema design because, unlike the relational model, it does not have normalisation and dependency theories to assist in database schema design. Second, the $O O$ approach uses inter-object references (through pointers) and the class hierarchy to represent relationships between classes. Different relationship types (m-n, n-ary, recursive etc.) are represented somewhat imperfectly. For example, consider the following schema from [13]:

(ob1, <name: "john", spouse: ob2>)

(ob2, <name: "mary", spouse: ob1>) 
where $o b 1$ and $o b 2$ are OIDs. There is an inverse relationship reference (a redundant relationship) in the spouse field that can introduce maintenance problems when there are changes in the spouse field, e.g. if "john" and "mary" are divorced, then references in the spouse field of both "john" and "mary" must be updated. This is a contradiction to the easy maintainability objective of the OO paradigm. To resolve this problem, the relationship between "john" and "mary" should be made explicit. As another example, consider the SUPPLIER (S), PART (P), SUPPLIES (SP) database [7], in which $S$ and $P$ are related by an m-n relationship type SP. Using inter-object references, each supplier object is associated with a set of part objects and their respective quantities. Such a structure does not facilitate symmetric queries. To circumvent this deficiency, each part object is then associated with a set of supplier objects and their respective quantities. This introduces redundancy which may lead to updating anomalies. The problems are similar to those of hierarchical models. These problems are amplified when $n$-ary $(n>2)$ relationships are considered. There is no feasible solution for representing an $n$-ary relationship using inter-object references in the $\mathrm{OO}$ paradigm.

Third, except for those OODBMSs that are based on the extended relational model (e.g. POSTGRES [21]), most OODBMSs do not fit into the 3-level schema architecture framework as spelled out in the ANSI/X3/SPARC proposal[2] for DBMSs. Users of most OODBMSs are often presented with a large-grained conceptual schema, with little or no facility for defining views. Some proposals for views in OODBMSs have been made[1,10,22], but the incorporation of a flexible and general view mechanism in OODBMSs is still a research issue.

Fourth, there can be inheritance conflicts in ISA hierarchies. Several techniques have been proposed to resolve these conflicts, e.g. choosing the first in a list of superclasses, as in ORION[4], using type information as in IRIS[8], denying the creation of (sub)classes with conflicting attributes, as in POSTGRES[21], and explicitly choosing the required property to inherit[8], as in $\mathrm{O} 2$ etc. These techniques are generally not satisfactory. For example, Orion's technique is quite arbitrary and may not yield the required semantics, while POSTGRES's approach is rather inflexible. It is not clear how IRIS determines type specificity. In $\mathrm{O} 2$, if two conflicting properties have different semantics, then explicitly choosing one of the two properties to inherit will preclude the user from inheriting the other property. This may not be desirable. In [19], we have proposed a complete algorithm which resolves inheritance conflicts by examining the semantics of the conflicting properties.

\section{An Object-Oriented Entity Relationship Diagram}

The ER approach for database schema design uses the concepts of entity type and relationship set, each of which has associated structural properties or attributes. An attribute can be single valued, multi-valued or composite. Different relationship sets are allowed, e.g. m-n, n-ary, recursive, existence dependent (EX) and identifier dependent (ID) weak relationship sets, special relationship 
sets such as ISA, UNION, INTERSECTION etc. The structure of a database can be represented by using an entity-relationship diagram.

Many extensions have been made to the ER approach to improve its usefulness as a database design tool. In [14], a normal form for ER diagrams was introduced which allows the 'goodness' of ER diagrams to be judged. Good normal form relations, either in $3 \mathrm{NF}$ or $5 \mathrm{NF}$, can be generated from a normal form ER diagram by using the techniques introduced in [14]. In [15, 16], the normal form ER diagram was used to represent the conceptual schema of a three level schema architecture. External schemas based on the hierarchical, network, ER and nested relations data models can be generated from the conceptual schema by using distinct sets of mapping rules. In effect, this approach allows a user to have different views of the normal form ER diagram.

We believe that this approach can be extended to support $O O$ external schemas, and to resolve the problems mentioned in Section 2. Specifically, all the structural properties of the $O O$ approach can be derived (generated) from an ER diagram[18]. For instance, an $O O$ class hierarchy can be directly represented using the ER relationship type 'ISA'. A composite object[11], e.g. a VEHICLE and its component parts, can be represented by the ER relationship type IS-PART-OF. Existentially dependent objects [12] which cannot exist independently of their parent objects correspond to ER weak entities. Each weak entity type is associated with a parent (regular) entity type through an existence dependent (EX) relationship or an identifier dependent (ID) relationship. The $\mathrm{OO}$ approach also supports the concept of a complex object. In a complex object, the relationships among component objects are not made explicit, but are embedded within the complex object as inter-object references. Note that a composite object is a complex object in which the relationships between an object and its component objects are IS-PART-OF relationships.: In the ER approach, the relationships among components of a complex object are made explicit. This is a strong advantage of the ER approach.

The ER model does not have the concept of methods. Furthermore, in the ER model, there are no concepts such as overloading and overriding of properties which are used in $O O$ inheritance hierarchies. These concepts can be incorporated in the ER approach, but inheritance conflicts can occur in ISA hierarchies and must be resolved.

We now define the notion of an OOER diagram, and then explain what we mean by inheritance conflicts in ISA hierarchies. In the rest of this paper, we une the term class to refer to entity type in order to conform to usual 00 notations.

Definition 1: An object-oriented entity relationship (OOER) diagram is an ER diagram augmented with methods and derived attributes. An OOER diagram is specifically characterised by the following:

- Methods and derived attributes can be defined for both entity types and relationship sets in an OOER diagram. We use the generic term properties to refer to methods, attributes and derived attributes. In an OOER diagram, methods and derived attributes are represented in the same way as attributes, except that they are suffixed by a pair of parentheses. A line end- 
ing in a single (double) arrowhead(s) links an entity type or relationship set to a method or derived attribute that returns a single (multiple) value(s), similar to the notation for single-valued (multi-valued) attributes. A line without arrowhead is used to link an entity type or relationship set to a method that performs some generally useful functions and does not return a value, e.g. printing an object's status, firing an employee etc; the cardinality information associated with arrowheads is meaningless in this context.

- Entity types related through the ISA relationship type are organised into an ISA hierarchy. Suppose A and B are entity types such that A ISA B. Then $A$ inherits, and possibly override, the attributes and methods of $B$.

- Components of complex objects belong to classes which are modelled as entity types in the OOER diagram. The relationship sets among these entity types are explicitly represented in the OOER diagram, using the usual notation for representing relationship sets in classical ER diagrams.

Figure 1 is an example OOER diagram which will be used to explain some of the concepts. In Figure 1, DOCTOR and NURSE both have a method called bonus() which returns a single value, while EMPLOYEE and PATIENT both have a derived attribute called age() which also returns a single value. PATIENT has a method called update(), which does not return a value. Note the line linking update() to PATIENT.

Definition 2: A property is specified in a class if it is either defined or redefined for the class. A redefined property overloads a similarly named property in some superclass(es) of the class.

As a general rule, only properties specified in an entity type (class) are represented in OOER diagrams. Sometimes, for clarity, we allow inherited key attributes to be explicitly represented for an entity type. For example, in Figure 1, empNo is explicitly represented in DOCTOR and NURSE even though empNo is specified in EMPLOYEE, and inherited by both DOCTOR and NURSE.

Definition 3: An inherited property is well-defined if it is specified in one and only one superclass, possibly indirect.

In Figure 2, method $\mathrm{p} 1()$ and attribute $\mathrm{p} 2$ are defined in class $\mathrm{Z}$. Method p1() is redefined in class $C$ and hence are explicitly represented as a property of class $\mathrm{C}$ in the OOER diagram. Class $\mathrm{B}$ inherits $\mathrm{p} 1()$ and $\mathrm{p} 2$ from class $\mathrm{Z}$, while class $\mathrm{C}$ inherits $\mathrm{p} 2$ from class $\mathrm{Z}$. Since these properties are inherited rather than specified in the classes, they are not explicitly shown on the OOER diagram.

Definition 4: A conflict situation exists when an inherited property is not well-defined, i.e. two or more superclasses specify the same property.

In Figure 2, classes B and C have commonly named properties p1() and p2, but only $\mathrm{p} 1$ () contributes to a conflict situation in class A. Method $\mathrm{p} 1()$ is not well defined in A (there are two classes $\mathrm{Z}$ and $\mathrm{C}$ which specify pl()), i.e. class $A$ has an inheritance conflict involving p1(), but p2 is well defined (only cless Z defines $\mathrm{p} 2)$.

In [19], we propose that conflicts occur because of: (1) redundant ISA relationships, (2) poor or erroneous design, (3) properties in superclases with the same name and same semantics, and (4) properties in superclasses with the 


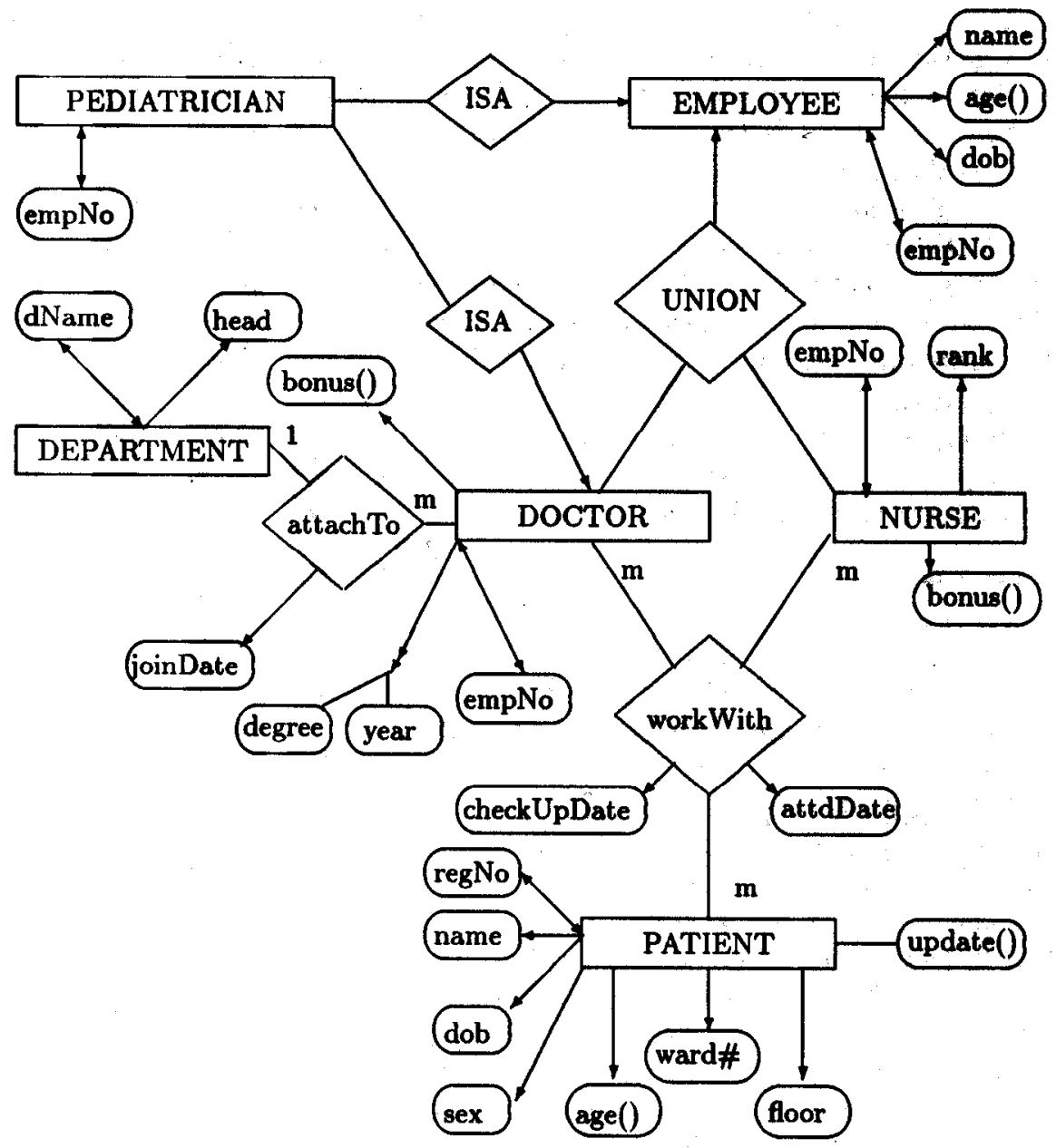

Fig. 1. An OOER diagram

same name and different semantics. A complete algorithm is proposed there which resolves inheritance conflicts systematically by considering the semantics of properties.

An OOER diagram may have undesirable redundancies and inheritance conflicts in its ISA hierarchies. For example, in Figure 1, suppose the functional dependency: ward\# $\rightarrow$ floor holds for PATIENT. Then, there will be redundancy if PATIENT records are stored based on the attributes for PATIENT shown in Figure 1. Furthermore, the dependency: ward\# $\rightarrow$ floor is not explicitly depicted on the OOER diagram. As another example, suppose CARING_DOCTOR is a subclass of both DOCTOR and NURSE in Figure 1. DOCTOR and NURSE both have a method bonus(). Then CARING_DOCTOR has a conflict situation which must be resolved. In the next section, a normal form for OOER diagram 


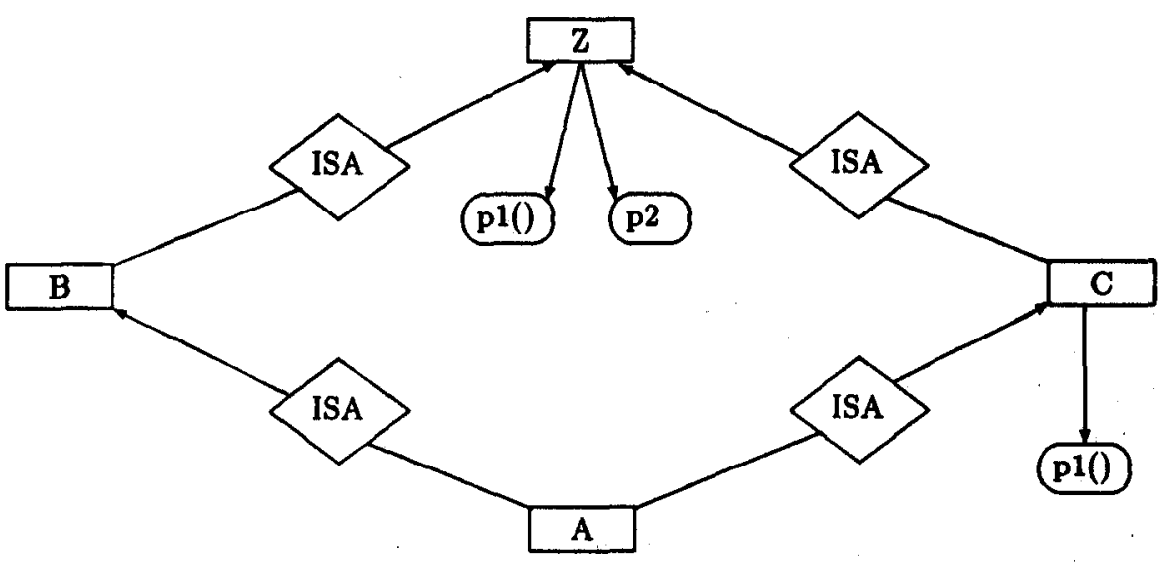

Fig. 2. An Inheritance Diagram

is defined that improves on an OOER diagram by removing undesirable redundancies and inheritance conflicts from ISA hierarchies.

\section{Normal Form OOER Diagram}

The objectives for defining a normal form OOER diagram from an OOER diagram are :

1. to capture and preserve the semantics of the real world, in terms of functional, multi-valued and join dependencies, by representing them explicitly in the OOER diagram.

2. to ensure that all the relationships represented in the OOER diagram are non-redundant, i.e. none of the relationships can be derived from other relationships.

3. to ensure that the relations translated from the ER diagram are in good normal form, either in $3 \mathrm{NF}$ or $5 \mathrm{NF}$.

4. to cnsure that all inheritance conflicts are eliminated from ISA hierarchies.

The concept of a normal form OOER diagram depends on the twin concepts of an entity type normal form and a relationship set normal form. Entity type and relationship set normal forms have been defined in [14]. The results there can be used directly because they improve on the structural properties of an OOER diagram and do not depend on the behavioural properties of the OOER diagram. We reproduce these results here for completeness. Note that definitions 5 to 10 and lemmas 1 to 4 are taken directly from [14], slightly modified to account for the concept of an OOER diagram. Definition 10 extends the original definition of a normal form for ER diagrams in [14]. 
Definition 5: Let $\mathrm{E}$ be an entity type and $\mathrm{K}$ be its identifier in an OOER diagram. The set of basic dependencies of $\mathrm{E}, \mathrm{BD}(\mathrm{E})$, is defined as follows:

1. For each many-to-one attribute $\mathrm{A}$ of $\mathrm{E}, K \rightarrow A$ is a functional dependency $(\mathrm{FD})$ in $\mathrm{BD}(\mathrm{E})$.

2. For each one-to-many multivalued attribute $\mathrm{A}$ of $\mathrm{E}, A \rightarrow K$ is an FD in $\mathrm{BD}(\mathrm{E})$.

3. For each one-to-many and many-to-many multivalued attribute $\mathbf{A}$ of $\mathbf{E}$, $K \rightarrow \rightarrow A$ is a multivalued dependency (MVD) in $\mathrm{BD}(\mathrm{E})$.

4. For each key $\mathrm{K} 1$ of $\mathrm{E}$ which is not the identifier of $\mathrm{E}, K \rightarrow K 1$ and $K 1 \rightarrow K$ are FDs in $\mathrm{BD}(\mathrm{E})$.

5. No other FDs or MVDs are in $\mathrm{BD}(\mathrm{E})$.

Informally, the set of basic dependencies of an entity type $\mathbf{E}$ are the functional dependencies and multivalued dependencies of $E$ which are explicitly shown in the OOER diagram.

Definition 6: An entity type $E$ of an OOER diagram is said to be in entity normal form (E-NF) if all functional dependencies and multivalued dependencies which only involve attributes of $E$, can be derived (or implied) from the set of basic dependencies of $\mathrm{E}, \mathrm{BD}(\mathrm{E})$, by using Armstrong's axioms for functional dependencies and inference rules for multivalued dependencies[5].

In Figure 1, suppose the FD: ward\# $\rightarrow$ floor holds for PATIENT. Then PATIENT is not in entity normal form because this dependency is not in, nor derivable from, the set of basic dependencies for the entity type. We normalise PATIENT in the next section.

Lemma 1: Let $E$ be an entity type of an OOER diagram. If $E$ is in E-NF, then the following statements hold:

1. Each single valued attribute $A$ of $E$ is fully dependent on each key of $E$ which does not contain $A$, and on each one-to-many attribute of $E$.

2. All components of any composite single valued attribute $A$ of $E$ are fully dependent on each key of $\mathbf{E}$ which does not contain $\mathbf{A}$.

3. There is no non-trivial functional dependencies defined among components of any composite attribute of $\mathbf{E}$.

4. For each one-to-many attribute $A$ of $E$ and for each many-to-many attribute $\mathrm{B}$ of $\mathrm{E}, A \rightarrow \rightarrow B$ is a strong MVD, i.e. the FD: $A \rightarrow B$ does not hold.

5. For each key $\mathrm{K}$ of $\mathrm{E}$ and for each multivalued attribute $\mathrm{A}$ of $\mathrm{E}, K \rightarrow \rightarrow A$ is a strong MVD.

6. No multivalued attribute of $\mathrm{E}$ is multi-dependent on a part of a key of $\mathrm{E}$.

7. No component of a composite multivalued attribute of $\mathrm{E}$ is multi-dependent on the identifier of $\mathbf{E}$.

Lemma 2: An entity type $\mathrm{E}$ of an OOER diagram is in E-NF if and only if it satisfies the following conditions:

1. Any non-trivial canonical form full dependency (i.e. the right gide of the FD is a single attribute) $A \rightarrow B$ which only involves attributes and components of composite attributes of $\mathbf{E}$ implies 
(a) $A$ is a key of $E$ or $A$ is a one-to-many attribute of $E$, and

(b) $\mathrm{B}$ is a single valued attribute or $\mathrm{B}$ is a component of a composite single valued attribute of $\mathbf{E}$.

2. Any strong MVD: $A \rightarrow \rightarrow B$ which only involves attributes and components of composite attributes of $E$ and in which $B$ is not multi-dependent on any proper subset of $A$ and no proper subset of $B$ is multi-dependent on $A$, implies

(a) $A$ is a key of $E$ or $A$ is a one-to-many attribute of $E$, and

(b) $\mathrm{B}$ is a multivalued attribute of $\mathrm{E}$.

Lemmas 1 and 2 can be proved directly using the definition of an E-NF entity type, Armstrong's axioms for functional dependencies and inference rules for multivalued dependencies.

Definition 7: Let $\mathrm{R}$ be a relationship set in an OOER diagram with identifier $\mathrm{K}$ and $\mathrm{F}$ be the associated set of functional dependencies which only involve the identifiers of the set of entity types participating in $R$. The set of basic dependencies of $R$, denoted by $B D(R)$, is defined as follows:

1. For each one-to-one attribute $\mathrm{A}$ of $\mathrm{R}, K \rightarrow A$ and $A \rightarrow K$ are FDs in $\mathrm{BD}(\mathrm{R})$.

2. For each many-to-one attribute $\mathrm{A}$ of $\mathrm{R}, K \rightarrow A$ is an $\mathrm{FD}$ in $\mathrm{BD}(\mathrm{R})$.

3. For each one-to-many multivalued attribute $A$ of $R, A \rightarrow K$ is an FD in $\mathrm{BD}(\mathrm{R})$.

4. For each one-to-many and many-to-many multivalued attribute $A$ of $\mathbf{R}$, $E \rightarrow \rightarrow A$ is an MVD in $\mathrm{BD}(\mathrm{R})$.

5. Let $A \rightarrow B$ be a full dependency in $\mathbf{F}$ such that $\mathbf{A}$ is a set of identifiers of entity types participating in $R$, and $B$ is the identifier of some entity type participating in $\mathrm{R}$. If $\mathrm{A}$ is a key of $\mathrm{R}$ or $\mathrm{B}$ is part of a key of $\mathrm{R}$ then $A \rightarrow B$ is an FD in $B D(R)$.

6. No other FDs or MVDs are in $\mathrm{BD}(\mathrm{R})$.

Definition 8: A relationship set $R$ of an OOER diagram is said to be in relationship normal form (R-NF) if all functional dependencies and multivalued dependencies which only involve attributes of $R$ and identifiers of entity types participating in $R$ are implied by the set of dependencies of $R$, i.e. $B D(R)$.

Informally speaking, the set of basic dependencies of a relationship set $\mathbf{R}$ includes those functional dependencies and multivalued dependencies which involve attributes of $R$ and are explicitly shown in the OOER diagram. Item 5 of the definition of the basic set of dependencies of a relationship set is to ensure that all relations which correspond to an R-NF relationship set are at least in 3NF.

In Figure 1, suppose the attribute attdDate of the relationship set workWith is functionally dependent on empNo of NURSE and regNo of PATIENT only. Then, workWith is not in relationship normal form. We normalise work With in the next section.

Lemma 3: Let $R$ be a regular relationship set in an OOER diagram and $B D(R)$ be the set of basic dependencies of $R$. If $R$ is in R-NF, then the following statements hold: 
1. All many-to-one attributes of $R$ are fully dependent on each key, each oneto-one attribute and each one-to-many attribute of $\mathbf{R}$.

2. All components of any composite single valued attribute $\mathbf{A}$ of $\mathbf{R}$ are fully dependent on each key of $R$, each one-to-many attribute and each one-toone attribute of $R$ which is not equal to $A$.

3. There is no non-trivial functional dependencies defined among components of any composite attribute of $R$.

4. For each one-to-many (or one-to-one) attribute $A$ of $R$ and for each manyto-many attribute $\mathrm{B}$ of $\mathrm{R}, A \rightarrow \rightarrow B$ is a strong MVD.

5. For each key $\mathrm{K}$ of $\mathrm{R}$ and for each multivalued attribute $\mathrm{A}$ of $\mathrm{R}, K \rightarrow \rightarrow A$ is a strong MVD.

6. No multivalued attribute of $R$ is multi-dependent on a part of a key of $\mathbf{E}$.

7. No component of a composite multivalued attribute of $R$ is multi-dependent on the identifier of $\mathrm{E}$.

This lemma is similar to lemma 1.

Lemma 4: Let $R$ be a regular relationship set in an OOER diagram and $B D(R)$ be the set of basic dependencies of $R$. $R$ is in R-NF if and only if it satisfies the following conditions:

1. Any non-trivial full dependency: $A \rightarrow B$ which only involves attributes, components of composite attributes, and identifiers of entity types participating in $R$, and in which $B$ is an attribute or a component of a composite attribute of $\mathrm{R}$ implies

(a) $A$ is a key of $R$, or $A$ is a one-to-many or one-to-one attribute of $R$, and

(b) $\mathrm{B}$ is a single valued attribute or a component of a composite single valued attribute of $R$.

2. Any non-trivial full dependency: $A \rightarrow B$ which only involves attributes of identifiers of entity types participating in $R$ and in which $B$ is a single attribute, implies either $A$ is a key of $R$ or $B$ is part of a key or $R$.

3. Any strong MVD: $A \rightarrow \rightarrow B$ which only involves attributes, components of composite attributes, and identifiers of entity types participating in $\mathbf{R}$ and in which $B$ is not multi-dependent on any proper subset of $A$ and no proper subset of $B$ is multi-dependent on $A$, implies

(a) A is a key of R, or A is a one-to-one or one-to-many attribute of $\mathbf{R}$, and

(b) $\mathrm{B}$ is a multivalued attribute of $\mathbf{R}$.

Lemma 4 is similar to lemma 2, except the extra condition (2) in lemma 4.

Definition 9: Let $D$ be an OOER diagram. The set of basic dependencies of $\mathrm{D}$, denoted by $\mathrm{BD}(\mathrm{D})$, is defined as the union of the sets of dependencies of all entity types of $D$ and the sets of basic dependencies of all relationship sets of D.

Definition 10: An OOER diagram $D$ is a normal form OOER diagram if it satisfies the following conditions:

1. All property names are distinct and of different semantics within an entity type or relationship set. All key attributes must be distinct, except for key attributes inherited from superclasses in ISA hierarchies. 
2. Every entity type in the OOER diagram is in E-NF.

3. Every relationship set in the OOER diagram is in R-NF.

4. All relationships and dependencies are implied by the set of basic dependencies of $\mathrm{D}$.

5. Every relationship set $R$ with no associated attribute defined on it, satisfies the condition that $R$ is not equal to a projection of the join of any two or more other relationship sets

6. There are no inheritance conflicts in its ISA hierarchies.

Informally, in condition one, we adopt a relaxed form of the universal relation assumption. The second condition ensures that all relations generated for all entity types are in 5NF. The fourth condition ensures that the OOER diagram has captured all the relationships and dependencies of the given database. The third and fifth conditions ensure that all relations generated for all regular relationship sets are either in $3 \mathrm{NF}$ or $5 \mathrm{NF}$ and that there is no relation in BCNF but not in $4 \mathrm{NF}$ or $5 \mathrm{NF}$.

\section{Deriving a normal form OOER diagram}

In this section, we convert the OOER diagram of Figure 1 to a normal form OOER diagram. The result of the conversion is shown in Figure 3. The following steps are adopted in order to achieve the conversion:

(Step 1) Ensure that all property names within each entity type and relationship set are distinct and of different semantics. Ensure that all key attributes are uniquely named, other than those inherited from superclasses.

(Step 2) Convert any non E-NF entity type to E-NF. We remove all undesirable functional dependencies and/or multivalued dependencies by introducing new entity types and relationship sets.

(Step 3) Convert any non R-NF relationship set to R-NF. We remove all undesirable functional dependencies, multivalued dependencies, and/or join dependencies either by introducing new entity types and relationship sets or by splitting the relationship set into smaller ones.

(Step 4) Remove each relationship set which has no associated attributes and is equal to a projection of the join of two or more other relationship sets.

(Step 5) Remove any inheritance conflicts from ISA hierarchies.

In step 1, we adopt a relaxed form of the universal relation assumption, as noted in Section 4, Definition 10. Key attributes must be unique, except for those inherited from superclasses. In Figure 3, for example, DOCTOR and NURSE inherits the key attribute empNo from EMPLOYEE. The empNo key attribute is therefore not uniquely named in this ISA (or UNION) hierarchy. Strictly, we need not display empNo of DOCTOR and NURSE explicitly on the OOER diagram, since empNo is specified in EMPLOYEE. Non-key attributes and method names may not be unique. For example, in Figure 3, both EMPLOYEE and PATIENT have similarly named attributes name and $d o b$, and derived attribute age(). In such cases, the context is sufficient to distinguish among these non-key attributes and methods. 


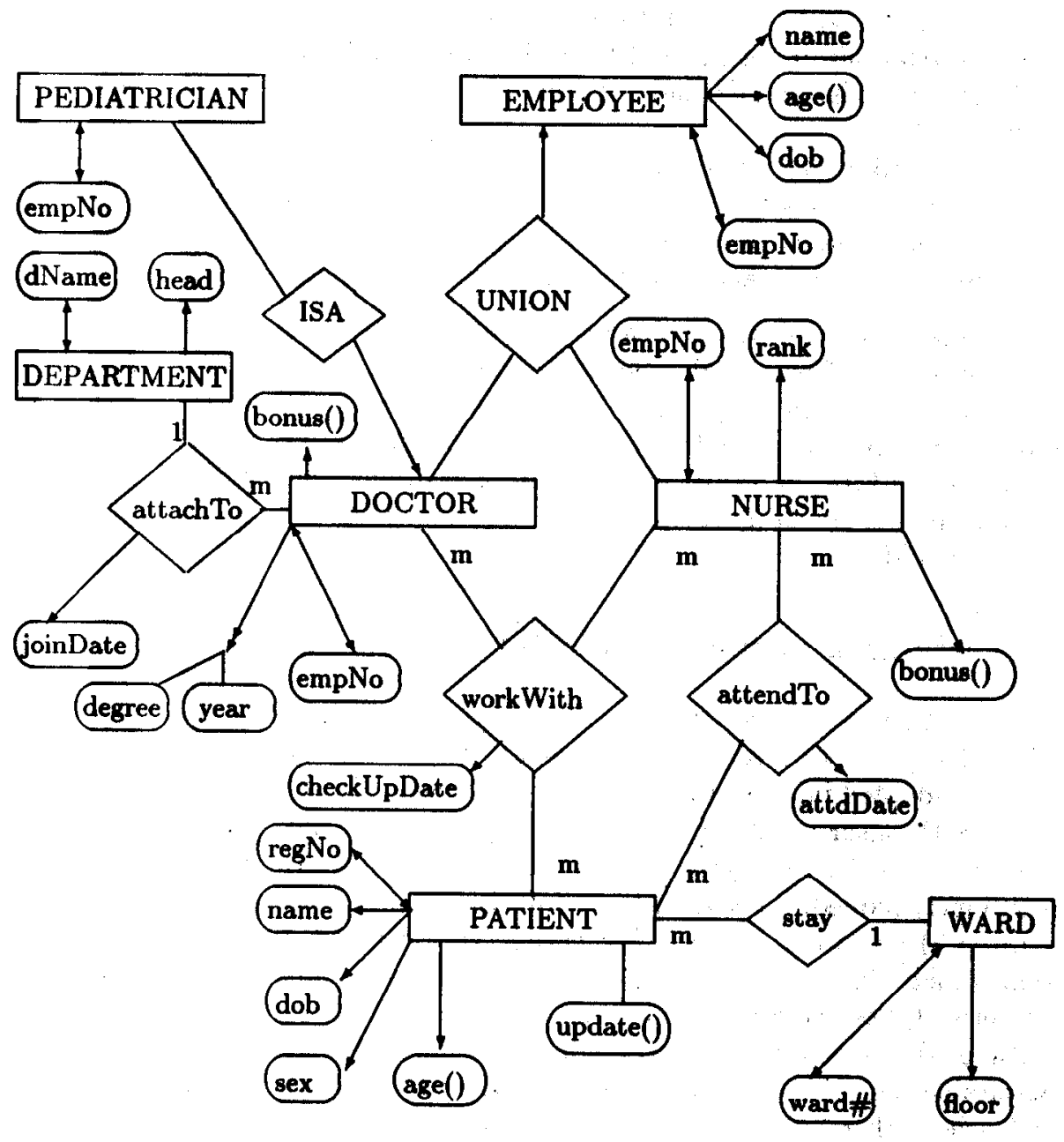

Fig. 3. Normal Form OOER Diagram

In Step 2, if an entity type is not in normal form, we remove some of the attributes involved and create some new entity types and relationship sets. For example, the PATIENT entity type in Figure 1 is normalised by removing its ward\# and floor attributes and creating a new entity type WARD and relationship set stay, as shown in Figure 3. This is to ensure that the FD: ward\# $\rightarrow$ floor is explicitly represented in the OOER diagram.

In Step 3, if a relationship set is not in normal form, we remove some of the attributes involved and create some new entity types and relationship rets, or split the relationship set into two or more smaller relationship sets. For example, in Figure 1, the relationship work With is not in relationship normal form because its attribute attdDate is functionally dependent on empNo of NURSE and regNo of PATIENT only. In Figure 3, we normalise the workWith relationship set of 
Figure 1 by creating a new relationship set attend To with an associated attribute attdDate.

In Step 4, we remove any relationship set with no associated attributes which is equal to a projection of the join of two or more other relationship sets. To detect such relationship sets, we require information about the semantic meaning of the relationship sets which can be provided by the database designer or owner. This step also removes any redundant ISA relationships. A direct ISA relationship A ISA C is redundant if there is some entity B such that A ISA B and B ISA C. This redundancy is a result of the transitivity of the ISA relationship set. In Figure 1, the ISA relationship set between PEDIATRICIAN and EMPLOYEE is redundant, and must be removed, as shown in Figure 3.

In Step 5, inheritance conflicts in ISA hierarchies are removed by using the method introduced in [19]. We will not describe the details here, but give an example to illustrate how we can remove inheritance conflicts.

Example: In Section 3, we describe a conflict situation involving a class CA RING_DOCTOR which is a subclass of both DOCTOR and NURSE (a multiple inheritance situation). DOCTOR and NURSE both specify a method bonus() which causes a conflict situation for CARING_DOCTOR. This conflict situation can be resolved by considering the semantics of bonus(). If the semantics (implementation) of bonus() is the same for both DOCTOR and NURSE, then it is better to factor bonus() to a more general class EMPLOYEE. If the semantics of bonus() is different, then several approaches can be taken to resolve the conflict[19]. First, we can rename the property bonus() for either DOCTOR or NURSE, or both, so that CARING_DOCTOR can inherit both these methods. This approach is not consistent with the use of similarly named properties in ISA hierarchies in order to support polymorphism. Second, CARING_DOCTOR may explicitly choose to inherit from either DOCTOR or NURSE, depending on the required semantics. This precludes CARING.DOCTOR from inheriting both bonus() methods. Third, CARING_DOCTOR may specify its own definition of the bonus() method, i.e. it overrides the definition of bonus() in its two superclasses.

We now explain how the normal form OOER diagram can be used to address three of the problems of Section 2. The problem related to views is addressed in the next section. Firstly, the normal form OOER diagram preserves application semantics by explicitly capturing entity types, relationship sets (m-n, n-ary, recursive, ISA etc.) and their attributes. By providing strong support for different relationship types among entity types, the ER approach eliminates the problems that the $O O$ approach faces in representing these relationship types. For instance, the problems of the 'spouse' relationship of Section 2, or the relationship between suppliers and parts (e.g. presence of redundant pointers, problems in supporting symmetric queries etc.) are eliminated.

Secondly, a normal form OOER diagram uses established ER, normalisation and dependency theories to provide a strong theoretical foundation. It is therefore possible to judge the quality of a schema based on the ER approach.

Thirdly, a normal form OOER diagram does not have any inheritance conflicts in its ISA hierarchies. This is by its definition. 


\section{Generating OO Schemas}

Each entity type in a normal form OOER diagram can be translated directly into a class of the $\mathrm{OO}$ schema. However, every translation of an $\mathrm{OO}$ schema from a normal form OOER diagram must handle relationship sets among entity types. Generally, three approaches can be adopted. The first two approaches depend on whether the underlying $O O$ data model of the $O O$ schema provides explicit support for the notion of relationship.

In approach one, the underlying 00 data model supports the notion of relationship directly. Each m-n, n-ary or recursive relationship set can be mapped dircctly into a class in the $O O$ schema. Pointers are then set up to link the relationship class to the classes participating in the relationship set. For example, we can define, using the $\mathrm{O} 2$ notation[8], classes for the NURSE entity type and the attendTo relationship set of Figure 3, as follows:

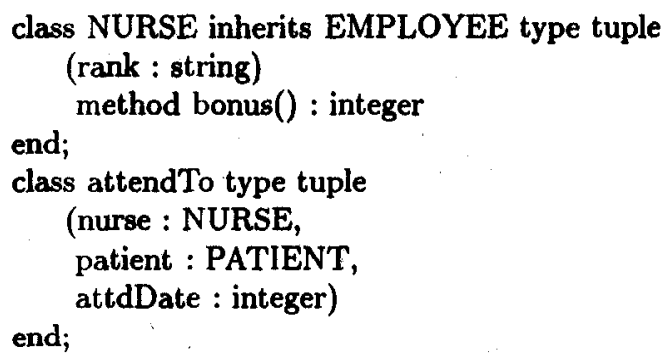

Note that EMPLOYEE and PATIENT are classes defined elsewhere, and that NURSE has a method bonus() that returns an integer value. Using approach one, each translated $\mathrm{OO}$ schema is of good quality. There are no undesirable redundancies because the schema is translated from a normal form OOER diagram.

Approach two provides for those $O O$ data models which do not support the notion of relationship. In this approach, the class definition for each entity type that participates in a relationship set with other entity types must have an embedded reference that refers to the participating entity types, and any attributes associated with the relationship set[18]. For example, the NURSE entity type of Figure 3 will have a tuple-valued attribute[18] that references a PATIENT class and the attdDate attribute associated with the relationship set attendTo, as follows (again using the $\mathrm{O} 2$ notation):

class NURSE inherits EMPLOYEE type tuple

(rank : string,

attendTo : set(tuple(patient : PATIENT, attdDate : string)), workWith : set(tuple(doc : DOCTOR, patient : PATIENT, checkUpDate : string)))

end;

method bonus() : integer 


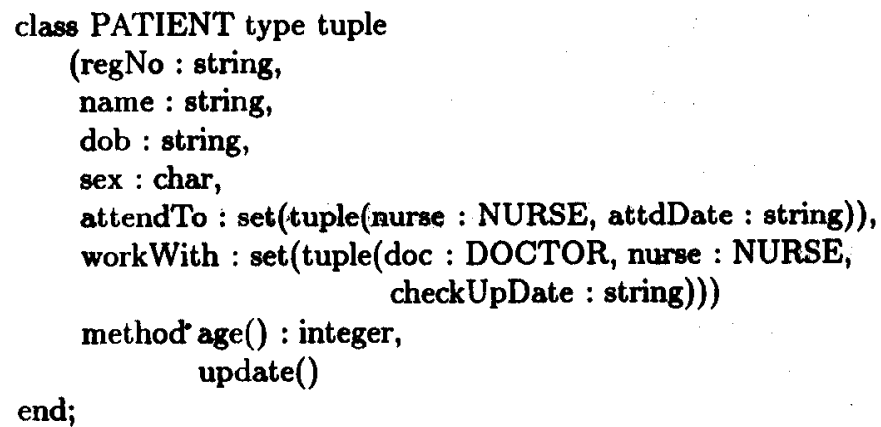

Note that EMPLOYEE is a class defined elsewhere and PATIENT has a method update() which does not return any value. Using approach two, each translated $O O$ schema does not contain undesirable redundancies except those arising from the existence of $m-n$ and $n$-ary relationship sets. These redundancies can be controlled at the system level, similar in concept to the physical/virtual pairing feature in IMS[7]. The spouse schema of Section 2 is another example of using approach two.

The third approach treats each 00 schema as a view of a normal form OOER diagram. We propose a three level ER-based schema architecture to support this view mechanism. The conceptual schema is represented by a normal form OOER diagram. Each $O O$ schema is a view or external schema of the conceptual schema that is generated by using a set of mapping rules. In [18], a set of mapping rules has been defined to generate $\mathrm{O} 2$ external schemas from an OOER diagram. This set of rules can still be applied to a normal form OOER diagram.

Each entity type in the conceptual schema can be mapped to a class in the OO external schema. If there is a relationship set between two entity types $E_{1}$ and $E_{2}$, then without loss of generality, $E_{2}$ can be a set-valued attribute in the class specification of $E_{1}$. For example, the class DOCTOR has an attribute DocPat whose value is a set of entities in the class PATIENT. Note that we can have different views of how the entity types are related in the external schema. For example, an instance of the class PATIENT can have a set of associated doctors while an instance of the class DOCTOR can have a set of associated patients. Any redundancy in the external schema is virtual as no redundancy exists in the conceptual schema.

The following $\mathrm{OO}$ external schema, again using the $\mathrm{O} 2$ notation, is generated by applying the mapping rules given in [18].

class EMPLOYEE type tuple

(empNo : string, name : string,

dob : integer)

end;

method age() : integer

class DOCTOR inherits EMPLOYEE type tuple

(qual : set(tuple(year : string, degree : string)),

Doc-Pat : set(PATIENT)) 
end;

method bonus() : integer

class NURSE inherits EMPLOYEE type tuple

(rank : string,

Nurse-Pat : set(tuple(patient : PATIENT, attdDate : integer)) ) $l^{*}$ vis attendTo $* /$

end;

method bonus() : integer

class PATIENT type tuple

(regNo : string,

name : string,

dob : string,

sex : char,

Pat-Doc : set(DOCTOR),

Pat-Nurse : set(NURSE)) /* via workWith */

method age() : integer,

update()

end;

Note that, in Figure 3, NURSE is related to PATIENT via two relationship sets, viz. attendTo and workWith. Therefore, there are two posaible derivations for the attribute Nurse-Pat in class NURSE and the attribute Pat-Nurse in class PATIENT. These two possibilities can be automatically generated by a schema design system and presented to the user for selection[17]. In this example, the user chooses to use the attendTo relationship set for deriving the NursePat attribute in NURSE, and the workWith relationship set for the.Pat-Nurse attribute in PATIENT. Note that it is also possible for the user to specify that Pat-Nurse is derived via attendTo, but with attdDate abetracted out.

The $O O$ external schema can include methods which have been defined in the conceptual schema. For example, the entity type EMPLOYEE has a method (derived attribute) called age() in the conceptual schema; this method can be included in the class EMPLOYEE in the external schema. This method age() is inherited by the classes DOCTOR and NURSE in the external scheme. Methods can also be specified in the external schema.

Notice that it is also possible for the user to define a view that computes the transitive closure of a relation, e.g. PART and its component parts. This can use the semi-naive or magic sets methods[3], but the exact implementation is transparent at the external schema level.

Sets of mapping rules can be defined to generate external schemas based on a number of data models (e.g. hierarchical, network, nested relations, relational, ER diagrams, etc.) from a conceptual schema that is represented by a normal form OOER diagram. In [15, 16], for example, mapping rules have been defined to generate external schemas based on hierarchical, network, relational, nested relations, and ER data models from a normal form ER diagram[14]. These rules are still valid for a normal form OOER diagram. The presence of methods and derived attributes in a normal form OOER diagram does not invalidate any of the guidelines in $[15,16]$. This is because the guidelines pertain to the 
structural properties of a normal form ER diagram, whereas methods and derived attributes provide an orthogonal behavioural perspective. Depending on the needs of users, the appropriate external schemas can be defined. External schemas may not necessarily be normalised, and therefore users may perceive some form of 'redundancy'. However, this redundancy is virtual in the sense that no redundancy exists at the conceptual schema level and data is not stored redundantly.

\section{Conclusion}

In this paper, we have defined the notion of an OOER diagram. An OOER diagram is a classical ER diagram augmented with methods and derived attributes. It incorporates $\mathrm{OO}$ concepts such as methods, inheritance (through the ISA, UNION, DFCOMPOSE, INTERSECTION relationships), class (entity or relationship), existentially dependent objects (weak entities) etc. An OOER diagram may have redundancies and inheritance conflicts in its. ISA hierarchies. The concept of a normal form OOER diagram is then proposed which improves on an OOER diagram by removing undesirable redundancies from the OOER diagram, and any inheritance conflicts from its ISA hierarchies. An OOER diagram is of 'good' quality if the OOER diagram is in normal form.

By using the normal form OOER diagram as a tool to design 00 schemas, we can address a number of $O O$ data modelling inadequacies, viz. (1) the inability to judge the quality of an $\mathrm{OO}$ database schema, (2) the presence of inheritance conflicts in ISA hierarchies, (3) the lack of explicit support for different types of relationship (m-n, n-ary, recursive etc.) in some 00 data models, and (4) the lack of flexible support for views. Object-oriented schemas can be translated from a normal form OOER diagram. Such translations must handle relationship sets that are explicitly represented in the normal form OOER diagram. We provide three different approaches to handle the translations. The first approach can be used to translate $O O$ schemas whose underlying $O O$ data models support the notion of relationship. Using this approach, the translated $O O$ schemas do not have any undesirable redundancies because they are translated from a normal form OOER diagram. The second approach is used when the underlying $O O$ data models do not support the notion of relationship. In this case, some redundancies may arise because of the existence of $m-n$ and $n$-ary relationship sets, but these redundancies can be controlled at the system level. The third approach provides a flexible view mechanism built on an ER-based 3-level schema architecture. In the third approach, the normal form OOER diagram is the conceptual schema while each $O O$ schema is a view or external schema that is generated from the conceptual schema by using a set of mapping rules. There may be redundancies at the external schema level, but these redundancies are virtual. No redundancies exist at the conceptual schema level. 


\section{References}

1. S. Abiteboul, A. Bonner: Objects and Views. Proc. ACM Sigmod Int. Conf. on Management of Data, 1991.

2. ANSI/X3/SPARC Study Group on Data Base Management Systems: Interim Report. FDT (ACM Sigmod bulletin) Vol. 7, No. 2, 1975.

3. F. Bancilhon, R. Ramakrishnan: An Amateur's Introduction to Recursive Query Processing. Proc. ACM Sigmod Int. Conf. on Management of Data, 1986, pp. 16-52.

4. J. Banerjee et al: Data model issues for object-oriented applications. ACM Transactions on Office Information Systems, Vol. 5, No. 1, Jan 87, pp. 3-26.

5. C. Beeri, R. Fagin, J. Howard: $A$ complete axiomatization for functional and multivalued dependencies in database relations. Proc. ACM Sigmod Int. Conf. Management of Data, 1977.

6. P. P. Chen: The entity-relationship model: towand a unified view of data. ACM Transactions on Database Systems, Vol. 1, No. 1, 1976.

7. C. Date: An introduction to database systems Vol. 1. Addison Wesley, 4th edition, 1986.

8. O. Deux et al: The 02 system. Communications of the ACM, Vol. 34, No. 10, Oct 1991, pp. 35-48.

9. D. Fishman et al: IRIS: An object-oriented database management system. ACM Transactions on Office Information Systems, Vol. 5, No. 1, Jan 87, pp. 48-69.

10. B.Hailpern, H.Ossher: Extending objects to support multiple interfaces and access controls. IEEE Transactions on Software Engineering, Vol. 16, No. 11, Nov 1990.

11. W. Kim et al: Composite object support in an object-oriented database system. Proc. OOPSLA, 1987.

12. W. Kim: An introduction to object-oriented databases. MIT Press, 1990.

13. C. Lecluse, P. Richard, F. Velez: O2, an object-oriented data model. Proc. ACM Sigmod Int. Conf. on Management of Data, Jun 1988.

14. T.W. Ling: A normal form for entity-relationship diagrams. Proc. 4th Int. Conf. on Entity-Relationship Approach, 1985, pp. 24-35.

15. T.W. Ling: $A$ three level schema architecture ER-based data base management system. Proc. 6th Int. Conf. on Entity Relationship Approach, 1987, pp. 181-196.

16. T.W. Ling: External schemas of entity-relationship based data base management systems. In Entity-Relationship Approach, C. Batini (Eds.), Elsevier Science Publishers, 1989.

17. T.W. Ling, M.L. Lee: A Prolog implementation of an entity-relationship based database management system. Proc. 10th Int. Conf. on Entity Relationship Approach, 1991.

18. T.W. Ling, P.K. Teo, L.L. Yan: Generating object-oriented views from an ERbased conceptual schema. 3rd Int. Symposium on Database Systems for Advanced Applications, Apr 6-8, Taejon, Korea, 1993.

19. T.W. Ling, P.K. Teo: Inheritance conflicts in object-oriented systems. Proc. Database and Expert Systems Applications, Prague, Czech Rep., Sep 1993.

20. T.W. Ling, P.K. Teo: Toward resolving inadequacies in object-oriented data models. Information and Software Technology, Vol. 35, No. 5, May 1993.

21. L. Rowe, M. Stonebraker: The Postgres data model. In The Postgres Papers, Memo UCB/ERL M86/85 (Revised), University of California, Berkeley, Jun 87.

22. J. Shilling, P. Sweeney: Three steps to views: extending the object-oriented paradigm. Proc. OOPSLA, 1989. 